\title{
Tools for Distributed Geodata Processing Based on OGC Web Processing Services
}

\author{
Felix Bensmann ${ }^{1}$, Dorian Alcacer-Labrador ${ }^{1}$, Rainer Roosmann ${ }^{1}$, Wassilios Kazakos ${ }^{2}$, \\ Roman Wössner ${ }^{2}$ and Andreas Abecker ${ }^{2}$ \\ ${ }^{1}$ University of Applied Sciences, Osnabrück/Germany · felix.bennsmann@hs-osnabrueck.de \\ ${ }^{2}$ Disy Informationssysteme GmbH, Karlsruhe/Germany
}

Short paper

\begin{abstract}
We sketch the main software components developed in the project RichWPS ${ }^{1}$, which aims to support end users (no IT experts) to make better use of distributed geodata processing through the OGC Web Processing Service (WPS). These services include a service orchestration solution (modelling GUI and orchestration engine), several supporting modules and services (like a semantics-enabled service registry as well as debugging and monitoring support for composed services), and some server-side helpers to facilitate true interoperability of services (like an adapter layer to bridge several "WPS dialects").
\end{abstract}

\section{Introduction}

While the OGC download and visualization services are extensively used by public administrations dealing with geo data, in particular for implementing the INSPIRE directive, the OGC Web Processing Service (WPS) ${ }^{2}$ is far less widely adopted. Although it seems obvious that processing services should be the next evolution step of service-based Spatial Data Infrastructures (SDIs), and that distributed geoprocessing has to offer manifold advantages, the practical usage - since years - is still mostly limited to academic prototypes. Besides non-technical barriers for a wider use of WPS in administrative practice (like the question of "payment" if one administrative unit develops and others re-use a WPS service), at least four significant technical problems of WPS were identified:

- Composition: lack of simple tools for building complex WPS processes from existing ones, comfortable enough for non-IT experts, including modelling support services like debugging or benchmarking.

- Retrieval: lack of comprehensive semantic metadata for better finding reusable components, including, e.g., QoS information.

- WPS dialects: data types and encoding of I/O parameters are not equal for all WPS implementations such that most WPS services are only interoperable if one specific cli-

1 The RichWPS project (2013-15) has been supported by the German Ministry for Education and Research (BMBF) within the "KMU-innovativ" programme under grant 01IS12041.

2 See http://www.opengeospatial.org/standards/wps

GI_Forum - Journal for Geographic Information Science, 1-2015.

(c) Herbert Wichmann Verlag, VDE VERLAG GMBH, Berlin/Offenbach. ISBN 978-3-87907-558-4.

(c) ÖAW Verlag, Wien. ISSN 2308-1708, doi:10.1553/giscience2015s16. 
ent-plus-server workbench is exclusively used; which means that there is in practice no cross-implementation interoperability.

- Output processing: the data-type agnostic protocol does not tell the client how to further process WPS results such that clients must either "know" the details of the reused WPS, or have no appropriate means for showing process results at the GUI.

We consider these issues serious impediments for true interoperability and reuse across organizational borders. Hence, the aim of the RichWPS research project ${ }^{3}$ was to design and prototypically develop methods and tools which support the non-IT expert end user in using WPS in order to foster the wider uptake of WPS technologies and remedy, to some extent, these shortcomings.

In former publications and talks, mainly the RichWPS case studies from environmental monitoring and marine-data management have been presented, and the RichWPS modeling and orchestration approach has been focused on. It was discussed that many existing approaches (like BPEL modelers and BPEL orchestration) were considered too much IToriented by the end users in the project - who are typically employees of the public administration with a domain-specific education like biology, geography, or civil engineering. It was also noted that existing modeling solutions lack modeling support like debugging or benchmarking tools for composed process models, or easily understandable functional descriptions for existing WPS processes. Hence, we thoroughly discussed a user-friendlier and more comfortable modeling and orchestration approach in ALCACER-LABRADOR et al. (2014), ZIEGENHAGEN et al. (2014), etc. In this paper, we focus on the client- and servercomponents for service execution not yet presented so far, which extend and complement the modeling and orchestration solution.

\section{RichWPS Overall Approach}

We sketch the interaction of the main components of the RichWPS solution (cf. ALCACERLABRADOR et al. 2014, ZIEGENHAGEN et al. 2014):

- The RichWPS ModelBuilder offers a GUI for domain experts for graphical modelling of complex processes from existing ones. The ModelBuilder automatically discovers existing processes and data through the SemanticProxy. The composed workflows can be tested, optimized and finally published and managed using the RichWPS Server.

- The RichWPS Server manages the centralized execution of workflows by an orchestration engine that interprets ROLA scripts. The RichWPS Orchestration Language ROLA has been developed as a custom language for OWS orchestration. The RichWPS server is an extension of the $52^{\circ}$ North WPS server ${ }^{4}$ with renovated functions for WPS lifecycle management (WPS-T based on SCHAEFFER (2008)). The RichWPS server also allows for testing and optimization of workflows by delivering intermediate results and profiling information to the ModelBuilder at design-time. Further, the RichWPS server provides information about the data types it works with and it supports the WPS Presentation Directives (HOFMANN et al. 2010).

\footnotetext{
3 See also http://richwps.github.io/

4 See http://52north.org/communities/geoprocessing/wps/
} 
- The RichWPS SemanticProxy is a catalog service, which realizes OWS service description and discovery. WPS or WFS services can be registered manually or discovered at runtime. Service metadata are stored in the Semantic Web language RDF (Resource Description Framework). Based on that, a custom vocabulary enables the interface- and content-specification beyond OWS service descriptions.

- The RichWPS Monitor enables the scheduled metering of WPS-services and contained processes. Based on prepared queries the Monitor can be used to deliver QoSinformation to the SemanticProxy. Linked systems, such as the ModelBuilder and the Server, are enabled to optimize workflows when needed.

These components that focus on the upper two issues listed above are complemented by client-side extensions dealing with the lower two issues in the list above.

\section{Client-Side Developments to Facilitate a WPS Ecosystem}

A number of further, mostly client-side, developments have been made for facilitating truly interoperable WPS-based infrastructures. Their usage is mostly not necessarily restricted to a purely RichWPS-based environment, but could support any WPS environment. These developments include:

- An agent-based intermediate communication layer for mobile WPS Clients, which aims at efficiently dealing with weak or unreliable internet connections.

- An Adapter Layer for WPS Clients that allows working with numerous widespread data types served by different WPS-server implementations including Esri ArcGIS Geoprocessing Services accessed through the ArcGIS REST interface (cp. Fig. 1).

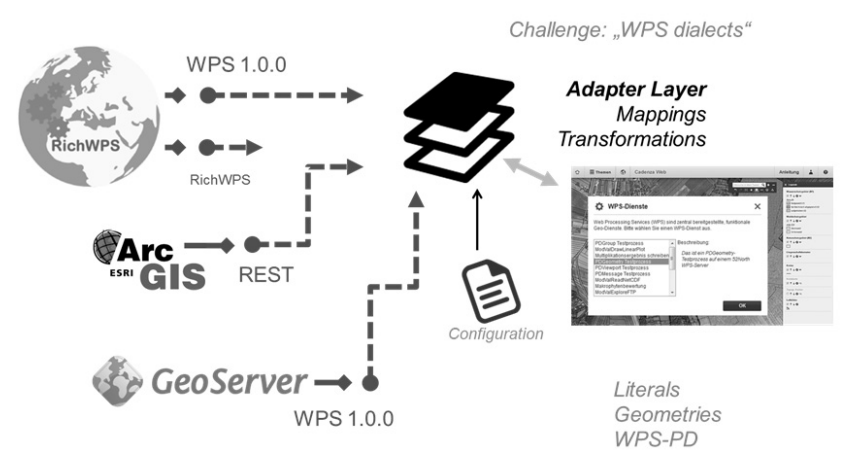

Fig. 1: Illustration of AdapterLayer for WPS clients to deal with WPS Dialects

- The inclusion of WFS layers in a WPS Client.

- A further-development and a prototypical implementation of client- and server-side (in the RichWPS server) for the Disy WPS Presentation Directives (WPS-PD, see HOFMANN et al. 2010) that allow the server to communicate to the WPS client the kind of WPS-result data types and the way how to present them in the GIS. 


\section{Conclusions and Outlook}

The software environment presented has been fully implemented and is partially available as Open Source on GITHUB. Two pilot applications have been realized in the context of the German Federal Waterways Engineering and Research Institute (BAW) and the Schleswig-Holstein Government-Owned Company for Coastal Protection, National Parks and Ocean Protection (LKN).

The RichWPS environment is not "yet another WPS workbench", but extends existing Open Source code (especially the $52^{\circ}$ North server and the Legato client) by elements not yet provided by other solutions. Parts of the solution could easily be integrated into other WPS implementations. RichWPS addresses the issues identified in the Introduction: (1) ModelBuilder, OrchestrationEngine and ROLA resolve the composition issue; (2) SemanticProxy shows a way how to address the retrieval issue; (3) the client-side AdapterLayer exemplarily solves part of the WPS dialect problem, in a generic and extensible manner; (4) output processing is facilitated through the WPS presentation Directives. We do not expect that these contributions are already sufficient to solve "all WPS problems". But we do think that besides the non-technical issues, WPS has significant inherent weaknesses and problems where the approaches presented could provide possible solutions. Based on practical experience, we also consider these problems (especially the interoperability wrt. data types of I/O parameters) severe blockers that must be eliminated before WPS can unfold its enormous application potential.

It should also be noted that - according to our knowledge regarding the upcoming WPS2.0 specification - although WPS2.0 will offer a couple of new and interesting features, we do not expect that it will address the problems of composition, WPS dialects, and output presentation.

\section{References}

Alcacer-Labrador, D., Bensmann, F. \& Roosmann, R. (2014), Web Processing Service (WPS) Orchestration - A Practical Approach. FOSS4G-E Academic Track Bremen.

HoFMANN, C. et al (2010), GDI-Dienste UIS BW - WPS-Dienste im Umweltinformationssystem Baden-Württemberg für die Geodateninfrastruktur Baden-Württemberg. F+EVorhaben KEWA - Kooperative Entwicklung wirtschaftlicher Anwendungen für Umwelt, Verkehr und benachbarte Bereiche in neuen Verwaltungsstrukturen Phase V 2009/10, R. MAYER-FÖLL et al. (Eds.), Karlsruhe: KIT Scientific Reports, 7544.

Schaeffer, B. (2008), Towards a Transactional Web Processing Service. GI-Days, Münster.

Ziegenhagen, D., Kohlus J. \& Roosmann, R. (2014), Orchestration of Geospatial Processes with RichWPS - A Practical Demonstration. In: EnviroInfo 2014: Proc. 28th Int. Conf. on Informatics for Environmental Protection, 525-532. 